\title{
The Effects of Eurycoma longifolia on Testosterone and Blood Pressure in High-Fat-Fed Animal Model
}

\author{
Rafidah Hanim Mokhtar ${ }^{1 *}$, Fakhria Al-Joufi ${ }^{2}$, Anil K Saxena ${ }^{3}$, Imad M Al-Ani ${ }^{3}$, Norlelawati A Talib ${ }^{3}$, \\ Norsidah Ku-Zaifah ${ }^{3}$ \\ ${ }^{1}$ Faculty of Medicine and Health Sciences, Universiti Sains Islam Malaysia, Nilai, Negeri Sembilan, Malaysia. ${ }^{2}$ College of Pharmacy, Al Jouf University, \\ Saudi Arabia. ${ }^{3}$ Kulliyyah of Medicine, International Islamic University Malaysia, Nilai, Negeri Sembilan, Malaysia.
}

\section{ARTICLE INFO}

Article history:

Received on: 14/09/2016

Accepted on: 09/03/2017

Available online: 30/04/2017

Key words:

Hypertension, Eurycoma

longifolia, Tongkat Ali,

Testosterone, Cardiovascular, Androgen.

\begin{abstract}
There is increased prevalence of hypertensives among Malaysians in the last decade. The cost of antihypertensive agents is also escalating. There is a dire need for cheaper alternative drugs. In tropical regions, there have been claims that Eurycoma longifolia (EL) a local herbal plant is effective in obliterating headache and reducing blood pressure. Most studies had focused on aphrodisiac property of EL which has been shown to enhance testosterone levels in males. This study exploredthe possible effect of EL as an anti-hypertensive agent and whether the mechanism is related to serum testosterone levels. Twenty four healthy male Sprague-Dawley rats were randomly divided into four groups $(n=6)$ : normal diet (ND), normal diet treated with EL (NDEL), high-fat diet (HFD) and HFD treated with EL (HFDEL). EL $(15 \mathrm{mg} / \mathrm{kg}$ ) was administered orally for 12 weeks. The animal's body weight, blood pressure and testosterone level were measured at week 0,6 and 12.Results showed that the level of testosterone in groups receiving EL were significantly increased $(\mathrm{P}<0.05)$ from the untreated groups, in agreement with previous studies report on EL increasing testosterone level. Rats with high fat diet benefitted from treatment with EL extract (HFDEL)as there were reduced systolic and diastolic blood pressure significantly $(\mathrm{P}<0.05)$. Despite this effect, there were no significant correlations between blood pressure and testosterone level in HFDEL. There were no changes of blood pressure in rats receiving normal diet with or without EL, but there was a significant negative correlation $(r=-0.846, p=0.034)$ between testosterone and diastolic blood pressure in NDEL. This study suggested the presence of anti-hypertensive property of EL in high fat diet induced hypertension, but it not associated with testosterone level. The blood pressure lowering mechanism through testosterone hormone however is only seen in normal diet group.
\end{abstract}

\section{INTRODUCTION}

Hypertension is one of thehealth problems with high morbidity and mortality rates in both developed and developing countries (Liang et al., 2014). The prevalence of essential hypertension among Malaysians aged 30 years and above has increased from $40.5 \%$ in 2004 (Rampal et al., 2008) to $43 \%$ in 2006 (Oteh et al., 2011). This is further complicated by the fact that $37.6 \%$ of diagnosed patients with hypertension were

\footnotetext{
* Corresponding Author

Rafidah Hanim Mokhtar, Faculty of Medicine and Health Sciences, Universiti Sains Islam Malaysia. Email: rafidahhanim @ usim.edu.my
}

unaware of their condition (Rashid and Azizah, 2011). The overall prevalence of essential hypertension was significantly higher in males $(29.6 \%)$ than in females $(26.0 \%)$ and increases progressively with age. Although hypertension has been known for over a century, its pathophysiology and management remain inconclusive. The health financing is also escalating and this includes the rising cost of anti-hypertensive agents. The levels of treatment and control remained to be inadequate (Lloyd-Sherlock et al., 2014). Eurycoma longifolia (EL) is aherbal medicinal plant of tropical region. In Malaysia and Indonesia it is popularly known as 'Tongkat Ali'. It belongs to the plant family Simaroubaceae. Alternative scientific names used for this shrub-tree include: Eurycoma merguensis, Picroxylon siamese, and Eurycoma longifolia Jack. 
Traditionally, it is marketed for its aphrodisiac property, however all parts of EL namely the barks and roots, have been used for a long time for the treatment of different diseases such as fever and headache apart from being used as a body tonic and antistress remedy (Zanoli et al., 2009). Unfortunately there has been lack of scientific evidence either in the form of laboratory experiments or clinical trials to support these claims.

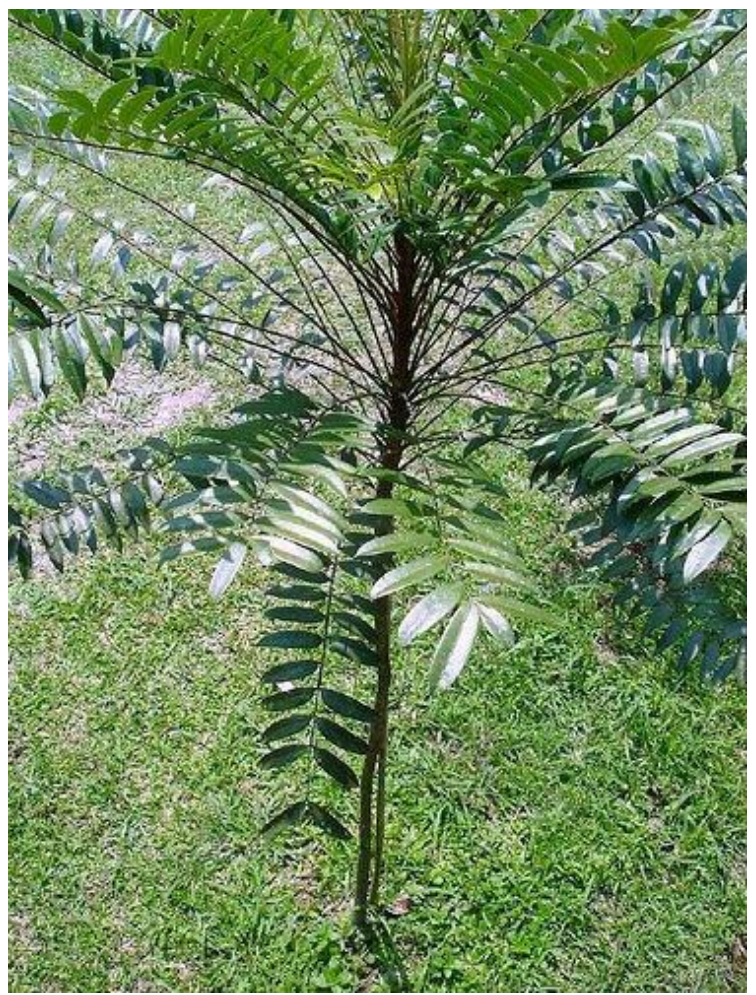

Figure 1.Eurycoma longifolia tree.

Botanically, it is a flowering, dioecous, un-branched and evergreen shrub that grows up to 10-15 meters in height (Figure 1). EL is subjected to many chemical and analytical studies. As technology progressed, a lot of useful compounds are fully isolated and their structures entirely elucidated.

Eurycoma longifolia has been shown to have antibacterial, antimalarial, antipyretic, antioxidant, cytotoxic, and anti-osteoporotic effects (Ang et al., 2003; Husen et al., 2004; Kardono et al., 1991; Mohd Ridzuan et al., 2007; Tada et al., 1991). Phytochemical studies on this plant revealed that parts of EL particularly the roots are rich with bioactive compounds such as quassinoids, squalene derivatives, and eurycolactones A-C (Ang et al., 2000).

The EL extract has exclusively been used for enhancing testosterone levels in males(Bhat and Karim, 2010). It has the ability to increase testosterone levels in the circulation of hypogonadic males and laboratory animals (Talbott et al., 2013). The quassinoids components in EL could positively influence men's cardiovascular disease (CVD) risk profile through enhancing testosterone levels. Many studies have investigated the roles of testosterone on blood pressure. Testosterone hormone changes markedly throughout the life of a man. Men with lower testosterone level have higher arterial blood pressure. This inverse relationship between testosterone level and arterial blood pressure suggests that hypertension in aging men is associated with decreased testosterone level (Sandberg and Ji, 2012). In a recent study done by Elena Passeri and his colleagues, they concluded that hypogonadism in males can be associated with some pathophysiological changes including severe bradycardia and heart failure (Passeri et al., 2014). Researchers have suggested the protective role of sex hormones (i.e. testosterone) in the aetiology of CVD by looking at the relationship between male gender and the prevalence of CVD (Morris and Channer, 2012).

The aim of the current study was to investigate whether EL is effective in reducing blood pressure through the mechanism involving testosterone.

\section{MATERIALS AND METHODS}

\section{Eurycoma longifolia (EL) Extract}

The EL extract powder PHYSTA®, was obtained from Biotropics Malaysia Berhad. In brief, the standardized aqueous extract was prepared by water extraction of EL roots and comprised high pressure water extraction followed by percolation, concentration by condensation, freeze drying and size reduction to obtain the dry extract powder. The dry extract powder of EL was standardized for contents are as shown in table 1.

Table 1: The standardized contents of Eurycoma longifolia extract (Physta $\left.{ }^{\circledR}\right)$.

\begin{tabular}{ccc}
\hline Test Parameter & Method/Reference & Specification \\
\hline Eurycomanone & HPLC & $0.8-1.5 \%$ \\
Total Protein & Lowry's Method & $>22 \%$ \\
Total Polysaccharide & Anthrone Reagent & $>30 \%$ \\
Glycosaponin & Gravimetric Method & $>40 \%$ \\
\hline
\end{tabular}

The aqueous extract was prepared by dissolving $15 \mathrm{mg}$ of EL extract in $10 \mathrm{ml}$ of distilled water.This allowed easy calculation of daily feeding dose according to the rat's body weight. The preparation was kept in refrigerator at temperature of $2-8 \mathrm{C}^{\mathrm{o}}$ and was removed from refrigerator 30 minutes prior to feeding to allow equilibrium to rat's body temperature(Tajul Ariff et al., 2012).

\section{High Fat Diet}

The compositions of high saturated fat diet are shown in table 2 .

Table 2: The composition of High Saturated Fat Diet.

\begin{tabular}{ll}
\hline INGREDIENT & AMOUNT \\
\hline Casein Purified High Nitrogen & $4000 \mathrm{gm}$. \\
DL-Methionine & $60 \mathrm{gm}$. \\
Sucrose & $6116 \mathrm{gm}$. \\
Corn Starch & $4000 \mathrm{gm}$. \\
Coconut Oil Hydrogenated & $4000 \mathrm{gm}$. \\
Alphacel, Non-Nutritive Bulk & $1000 \mathrm{gm}$. \\
DL-a-Tocopherol Powder (250 IU/gm.) & $24 \mathrm{gm}$. \\
AIN-76 Mineral Mix & $800 \mathrm{gm}$. \\
Plus MP Vitamin Diet Fortification Mixture 1.2 x Normal & \\
\hline
\end{tabular}




\section{Animals and experimental design}

Twenty four young, adult male Sprague Dawley (SD) rats weighing $250-300 \mathrm{~g}$ were housed in standard plastic cages (2 rats per cage). They were maintained at room temperature (22$24^{\circ} \mathrm{C}$ ) with adequate ventilation, $12 \mathrm{~h}$ light-dark cycle and about $(50 \pm 5 \%)$ humidity. After one week of acclimatization, they were randomly divided into four groups of 6 animals each and treated for 12 weeks as follow: Group ND was given only normal diet, group NDEL was given normal diet and EL extracts $(15 \mathrm{mg} / \mathrm{kg})$ dissolved in distilled water, group HFD was given only high fat diet and group HFDEL was given high fat diet and EL extracts $(15 \mathrm{mg} / \mathrm{kg})$.

The EL extract was administered by gastric gavage. At the end of $12^{\text {th }}$ week, the rats were kept in fasted state for 12 hours prior to anaesthesia and then sacrificed by cervical dislocation.

\section{Blood Pressure Measurement}

The blood pressure was measured and analyzed on 3 occasions, at week 0,6 and 12 by using CODA® tail-cuff blood pressure system which utilized volume pressure recording (VPR) sensor technology. Baseline systolic blood pressure (SBP) and diastolic blood pressure (DBP) were recorded at week 0. The measurement of SBP and DBP were repeated at week 6 and 12 to assess the effects of EL on BP.

In brief, the rat was trained and acclimated in the animal holder for 15 minutes each day for three consecutive days before beginning the experiment.

This help to create a low-stress environment and obtain accurate and consistent BP measurements. The rat was allowed to enter the holder (restrainer) freely and nose cone was used to prevent excessive animal movement during BP testing. The occlusion cuff was threaded through the tail and secured in the notch at the top rear of the holder.

\section{Blood Sampling}

Blood samples were collected from all experimental groups under general anesthesia; the diethyl ether. A capillary tube was gently inserted in the optical sinus and the required volume of blood was collected in a sterile glass tube containing gel for serum separation. After allowing the blood to clot at room temperature for 20-30 minutes the blood samples were centrifuged at $3000 \mathrm{rpm}$ for 15 minutes. The centrifuged serum was stored at $-80^{\circ} \mathrm{C}$ until the biochemical evaluation was done.

\section{Serum lipid assay}

Serum total cholesterol and high density lipoprotein cholesterol (HDL-c) were determined using Randox Laboratory kit reagents. Serum triglyceride level was estimated using Randox Laboratory test kit. Low density lipoprotein cholesterol (LDL-c) was estimated indirectly from the measured levels of triglyceride (TG), high-density lipoprotein cholesterol (HDL-c), and total cholesterol (TC) using the Friedewald equation:
$\mathrm{LDL}=\mathrm{TC}-\mathrm{HDL}-(\mathrm{TG} / 2.17)$ (Friedewald, Levy and Fredrickson, 1972).

\section{Testosterone Assay}

Testosterone levels were measured in serum $(\mathrm{nmol} / \mathrm{L})$ using BioVision's Testosterone EIA kit (cat. \#K7418-100). It operates on the basis of competition between the hormone conjugate and the testosterone in the serum for a limited number of binding sites on the antibody coated plate. Quantitative test results were obtained by measuring and comparing the absorbance reading of the wells of the samples against the standards with a microplate reader at $450 \mathrm{~nm}$.

\section{Ethics in Research}

The animals were treated according to the Standards and Regulations for the Care and Use of Laboratory Animals of the National Institutes of the Health and according to the guidelines of IIUM animal Ethical Committee, the reference number (IIUM/519/14/4/IACUC).

\section{Statistical Analyses}

The statistical software SPSS 22.0 was used. All analyses were conducted using one-way and repeated measures ANOVA. Post-hoc comparison (Tuky's test) was employed to assess the overall significance among groups. Correlations between total testosterone level and IMT were calculated using Pearson's correlation coefficient. P value less than 0.05 was considered statistically significant.

\section{RESULTS}

There was no significant difference in the mean weight of diet consumption per week among all groups. The control group (ND) had ingested a mean amount of $33 \pm 2$ gram food per day $(\mathrm{g} / \mathrm{d})$, while other groups ingested $32 \pm 2 \mathrm{~g} / \mathrm{d}(\mathrm{p}>0.05, \mathrm{n}=6$ per group).

There were no significant body weight changes in experimental groups as compared to rats with normal diet. NDEL however has significantly reduced bodyweight as compared to the HFD group (refer Table 3).

Table 3: Percentage Changes in the Body Weight of Experimental Groups

\begin{tabular}{lcccc}
\hline \multicolumn{1}{c}{ Body Weight (BW) } & ND & NDEL & HFD & HFDEL \\
\hline Initial BW at week 0 (gm) & $293 \pm 36$ & $279 \pm 29$ & $274 \pm 33$ & $276 \pm 36$ \\
Final BW at week 12 (gm) & $395 \pm 45$ & $338 \pm 38$ & $374 \pm 33$ & $343 \pm 45$ \\
Changes of BW by (\%) & $34 \% \pm 7$ & $21 \% \pm 7^{\#}$ & $37 \% \pm 13$ & $25 \% \pm 7$ \\
\hline The values are mean \pm SD. (n=6) & & & & \\
${ }^{\#} \mathrm{p}=0.025$ as compared to HFD. & & & &
\end{tabular}

\section{Effects of Eurycoma longifolia on Blood Pressure}

There were significant reductions of SBP and DBP in week 6 and week 12 in HFDEL group as compared to HFD group. EL has no significant effect in rats receiving normal diet as compared to untreated rats (refer Table 4). 
Table 4: Effect of EL on Systolic and Diastolic BP (mmHg) of all rats from different groups.

\begin{tabular}{cccccc}
\hline \multirow{2}{*}{ Parameters } & & ND & NDEL & HFD & HFDEL \\
\hline \multirow{2}{*}{ Week 0 } & Sys. & $99.67 \pm 8.9$ & $98.33 \pm 7.3$ & $97.2 \pm 3.97$ & $100.5 \pm 5.54$ \\
& Dia. & $73.8 \pm 4.3$ & $71.8 \pm 4.1$ & $76.8 \pm 6.6$ & $73.5 \pm 5.2$ \\
\hline \multirow{2}{*}{ Week 6 } & Sys. & $98.83 \pm 11.1$ & $101.83 \pm 2.7$ & $144 \pm 7.01^{*}$ & $121.2 \pm 14.1^{* \#}$ \\
& Dia. & $73.3 \pm 5.4$ & $79.8 \pm 7.7$ & $105.5 \pm 6.8^{*}$ & $93.17 \pm 10.3^{* \#}$ \\
\hline \multirow{2}{*}{ Week 12 } & Sys. & $95 \pm 3.03$ & $99 \pm 4.94$ & $155 \pm 18.01^{*}$ & $131.8 \pm 4.8^{* \#}$ \\
& Dia. & $72.3 \pm 8.6$ & $79.3 \pm 8.6$ & $130.5 \pm 14.2^{*}$ & $103.2 \pm 11.02^{* \#}$ \\
\hline
\end{tabular}

The values are mean $\pm \mathrm{SD}(\mathrm{n}=6)$.

$* \mathrm{P}<0.05$ as compared to ND.

${ }^{\#} \mathrm{P}<0.05$ as compared to HFD.

Table 5: Descriptive Statistics of Total Cholesterol at Week 0, 6 and 12.

\begin{tabular}{|c|c|c|c|c|c|}
\hline \multirow{2}{*}{\multicolumn{2}{|c|}{ PARAMETERS }} & \multicolumn{4}{|c|}{ GROUPS } \\
\hline & & ND & NDEL & HFD & HFDEL \\
\hline \multirow{4}{*}{ 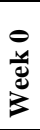 } & TC & $0.9 \pm 0.3$ & $0.8 \pm 0.4$ & $0.8 \pm 0.3$ & $0.8 \pm 0.3$ \\
\hline & TG & $0.55 \pm 0.2$ & $0.51 \pm 0.4$ & $0.55 \pm 0.27$ & $0.59 \pm 0.28$ \\
\hline & LDL & $0.43 \pm 12$ & $0.39 \pm 0.2$ & $0.30 \pm 0.14$ & $0.38 \pm 0.2$ \\
\hline & HDL & $0.21 \pm 0.1$ & $0.23 \pm 0.14$ & $0.23 \pm 0.11$ & $0.18 \pm 0.1$ \\
\hline \multirow{4}{*}{ 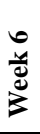 } & TC & $1 \pm 0.2$ & $0.9 \pm 0.2$ & $1.3 \pm 0.3$ & $1.2 \pm 0.2$ \\
\hline & TG & $0.7 \pm(0.2$ & $0.65 \pm 0.2$ & $1.22 \pm 0.2$ & $0.84 \pm 0.23$ \\
\hline & LDL & $0.45 \pm 0.2$ & $0.39 \pm 0.2$ & $0.45 \pm 0.3$ & $0.45 \pm 0.3$ \\
\hline & HDL & $0.25 \pm 0.1$ & $0.23 \pm 0.1$ & $0.32 \pm 0.2$ & $0.3 \pm 0.1$ \\
\hline \multirow{4}{*}{ 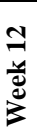 } & TC & $0.9 \pm 0.2$ & $0.55 \pm 0.3$ & $1.4 \pm 0.2 *$ & $1 \pm 0.3^{*}$ \\
\hline & TG & $0.5 \pm 0.2$ & $0.25 \pm 0.1$ & $1.5 \pm 0.9 *$ & $0.79 \pm 0.3 \dagger$ \\
\hline & LDL & $0.44 \pm 0.2$ & $0.34 \pm 0.1$ & $0.62 \pm 0.24$ & $0.38 \pm 0.1$ \\
\hline & HDL & $0.21 \pm 0.1$ & $0.19 \pm 0.2$ & $0.3 \pm 0.1$ & $0.3 \pm 0.1$ \\
\hline
\end{tabular}

The values are means $\pm \mathrm{SD} ;(n=6)$.

$* \mathrm{P}<0.05$ as compared to ND.

$\dagger \mathrm{P}<0.05$ as compared to HFD.

$\mathrm{TC}=$ Total Cholesterol, TG=triglyceride, $\mathrm{LDL}=$ Low density lipoprotein, HDL=high density lipoprotein.

\section{Effects of Eurycoma longifolia on Serum Lipid Profile}

Lipid profile levels of all experimental groups were evaluated and showed in table 5. There were significantly increases in TC and TG in both groups (HFD and HFDEL) as compared to ND group. On the other hand, there was no significant change ( $p>0.05)$ in lipid profile parameters of NDEL when compared to ND. HFDEL rats have high significant decrease $(\mathrm{P}<0.05)$ in its TG levels as compare to HFD group. There were no significant differences in TC, HDL-C and LDL-C in HFDEL compared to HFD . There was no significant changes in HDL-c between the treated groups and untreated groups $(\mathrm{P}>0.05)$.

\section{Effects of Eurycoma longifolia on Testosterone level}

At week 0 , the testosterone levels were not significantly different in all experimental groups. At week 6, the level of testosterone in both treated groups fed with were increased ( $\mathrm{p}$ $<0.05$ ) from their comparative groups (ND vs. NDEL, $\mathrm{p}<0.05$ ) and (HFD vs. HFDEL, $\mathrm{p}<0.05$ ) (Table 6). The observed $\mathrm{F}$ value was statistically significant $(\mathrm{F}(3,20)=52.3, \mathrm{p}<0.001))$, partial $\eta 2=0.89$, which indicated there was a difference of testosterone levels among groups over time.

Figure 2 shows the graph of mean serum testosterone levels at week 0,6 and 12 for each group. The increasing trend in both treated groups continued till week 12 . The mean testosterone level of the NDEL group increased from $3.017(0.36)(\mathrm{nmol} / \mathrm{l})$ to $8.3(1.2)(\mathrm{nmol} / \mathrm{l})$ and increased from $3.87(0.62)(\mathrm{nmol} / \mathrm{l})$ to 8.13 (1.2) $(\mathrm{nmol} / \mathrm{l})$ in HFDEL group. On the other hand, the mean of testosterone levels in ND and HFD were almost equal throughout the study period.

Table 6: Serum testosterone levels (in nmol/l; mean \pm SD) in studied groups at 0,6 and 12 week.

\begin{tabular}{lllll}
\hline Groups Weeks & \multicolumn{1}{c}{ ND } & \multicolumn{1}{c}{ NDEL } & \multicolumn{1}{c}{ HFD } & \multicolumn{1}{c}{ HFDEL } \\
\hline $\mathbf{0}$ & $3 \pm 1$ & $3.02 \pm 0.4$ & $3.3 \pm 1.3$ & $3.87 \pm 0.6$ \\
$\mathbf{6}$ & $3.2 \pm 0.8$ & $8.4 \pm 1.2^{*}$ & $3.4 \pm 1.02$ & $8.13 \pm 1.2^{*} \dagger$ \\
$\mathbf{1 2}$ & $4 \pm 0.98$ & $23.8 \pm 6.8^{*}$ & $3.43 \pm 0.98$ & $19.3 \pm 4 * \dagger$ \\
\hline
\end{tabular}

The values are mean \pm SD $(n=6)$

$* \mathrm{P}<0.05$ as compared to ND.

$\dagger \mathrm{P}<0.05$ as compared to HFD.

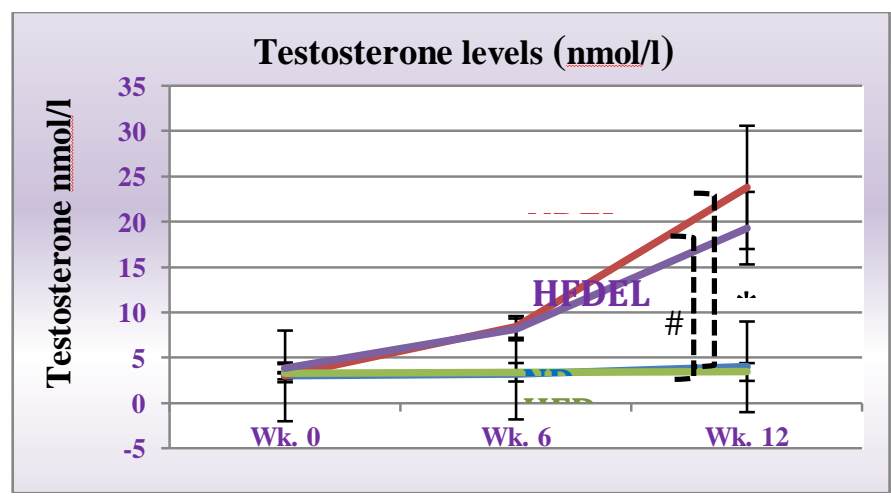

Figure 2.The values are mean $\pm S D ;(n=6) . * P<0.05$ as compared to ND. \# $\mathrm{P}<0.05$ as compared to HFD. 


\section{Correlation analysis of blood pressure and testosterone level}

To assess the correlation of testosterone with blood pressure, Pearson's correlations analysis was performed (Table 7). For systolic BP analysis, there were weak negative correlations of between systolic BP and testosterone level in all groups except in NDEL which showed weak positive correlations. However they did not reach statistically significant level. In diastolic BP analysis, there was a significantly strong negative correlation $(r=-$ $0.846, \mathrm{p}=0.034$ ) between the testosterone level in NDEL. The rest of the group showed weak negative correlations between diastolic BP and testosterone level and did not reach a statistically significant level.

Table 7: Correlation of Testosterone with Blood Pressure

\begin{tabular}{cccc}
\hline & $\begin{array}{c}\text { Correlation } \\
\text { with }\end{array}$ & Pearson correlation & \multirow{2}{*}{ P-value } \\
\hline \multirow{2}{*}{ ND Group } & Systolic BP & -0.689 & 0.13 \\
& Diastolic BP & -0.199 & 0.71 \\
\hline \multirow{2}{*}{ NDEL Group } & Systolic BP & 0.156 & 0.77 \\
& Diastolic BP & -0.846 & $0.034^{*}$ \\
\hline \multirow{2}{*}{ HFD Group } & Systolic BP & -0.399 & 0.43 \\
& Diastolic BP & -0.6 & 0.21 \\
\hline \multirow{2}{*}{ HFDEL Group } & Systolic BP & -0.408 & 0.42 \\
& Diastolic BP & -0.125 & 0.81 \\
\hline
\end{tabular}

Correlations is significant at $\mathrm{P}<0.05$

Correlations is highly significant at $\mathrm{P}<0.01$

\section{DISCUSSION}

Eurycoma longifolia (EL) or Tongkat Ali is receiving the attention in recent years because of the beneficial effects it claims to have on many health problems and diseases, apart from being readily available and has been popularly commercialized as beverages with aphrodisiac properties.

High fat diet in this study contributed to the elevation of blood pressure in rats as compared to the normal diet group. In vivo findings confirmed that daily administering of EL reduced the systolic and diastolic BP in rats with high fat diet (HFDEL) as early as from 6 weeks of EL treatment. This anti-hypertensive property of EL however did not affect the blood pressure in groups which was not fed with high fat diet (NDEL) in which their blood pressure remained stable during the 12-week term treatment.

Similarly, after six weeks, the groups receiving EL (NDEL and HFDEL) showed significant rises in testosterone levels in comparison with the untreated groups (ND and HFD). This was supported by previous study where EL enhances testosterone levels in intact rats (Zanoli et al., 2009; Saadiah Abdul Razak et al., 2012; Tajul Ariff et al., 2012; Low et al., 2013). When both groups treated with EL was compared with each other (NDEL and HFDEL), there was no significant differences of testosterone levelafter dosing the male rats with $15 \mathrm{mg} / \mathrm{kg}$ of EL, as this is attributable to the beneficial effect of EL itself but not to the differences in fat content of the diet, thus providing further evidence of the folk-use of EL as aphrodisiac and testosterone booster. This was consistent with reports from previous studies on phytoandrogenic effect of EL in rats (George \& Henkel, 2014). Though the aphrodisiac effect of EL has been extensively studied, the mechanisms underlying this effect remain far less clear. One of the proposed mechanisms is that EL has the ability to activate CYP17, steroid 17-alpha-monooxygenase, which catalyzes the last step of androgen biosynthesis in testes (Tajul Ariff et al., 2012).

In correlation analysis, only NDEL group showed significant inverse relationship between testosterone level and diastolic blood pressure. Androgens in particular testosterone has been shown to play vital role in vasculature system through androgen receptors which are present in endothelial cells. Animal studies have consistently demonstrated that testosterone is atheroprotective, whereas testosterone deficiency promotes the early stages of atherogenesis (Kelly and Jones, 2013). Hall et al(2011) concluded that testosterone, at physiological concentrations, can selectively suppress $\mathrm{Ca} 2+$ entry into A7r5 cells via L-type $\mathrm{Ca} 2+$ channels thus it is a likely mechanism underlying its vasodilatory actions (Hall et al., 2011). Based upon the results of the present study, EL may increase the testosterone levels and decreased the high blood pressure via androgen receptors but without the presence of other co-morbid factor such as high serum lipid.

\section{CONCLUSION}

The results of the current study demonstrated that EL is a testosterone booster and has a potential protective effect against high blood pressure which is induced by high fat diet. However the mechanism of how blood pressure is reduced in high fat diet groups seemed to be in other pathway other than testosterone mechanism. The role of testosterone in reducing $\mathrm{BP}$ is more pronounced in normal diet and normal lipid profile rats. Based on the results, further study is needed to evaluate the exact role of EL as a hypotensive agent.

\section{ACKNOWLEDGEMENT}

Authors would like to thank USIM for sponsoring and supporting this research study (USIM/PPP/PSK-02-12411). We warmly thank all the staff of pharmacology lab and Molecular Research Laboratory department Kulliyyah of Medicine, International Islamic University of Malaysia for their help and skillful technical assistance.

\section{Financial support and sponsorship: Nil.}

Conflict of Interests: There are no conflicts of interest.

\section{REFERENCES}

Ang HH, Hitotsuyanagi Y, Takeya K. Eurycolactones A-C, novel quassinoids from Eurycoma longifolia, Tetrahedron Lett., 2000; 41: 6849-6853.

Ang HH, Ngai TH and Tan TH. Effects of Eurycoma longifolia Jack on sexual qualities in middle aged male rats, Phytomedicine., 2003; 10(6): 590-593.

Bhat R, Karim AA. Tongkat Ali (Eurycoma longifolia Jack): A review on its ethnobotany and pharmacological importance, Fitoterapia., 2010; 81(7): 669-679. 
Friedewald, W.T., Levy, R.I. \& Fredrickson, D.S. Estimation of the concentration of low-density lipoprotein cholesterol in plasma, without the use of the preparative ultracentrifuge.Clinical Chemistry.,(1972); 18: 499-502.

Hall J, Jones RD, Jones TH, Channer KS and Peers C. Selective Inhibition of L-Type Ca2+ Channels in A7r5 Cells by Physiological Levels of Testosterone, Endocrine Society., 2011; 147(6): 2675-2680

Husen RI, Pihie AH, Nallappan M. Screening for antihyperglycaemic activity in several local herbs of Malaysia., $J$ Ethnopharmacol., 2004; 95(3) : 205-208.

Kardono LB, Angerhofer CK, Tsauri S, Padmawinata K, Pezzuto JM and Kinghorn AD. Cytotoxic and Antimalarial Constituents of the Roots of Eurycoma longifolia, J Nat Prod., 1991; 54(5): 1360-1367.

Kelly DM, Jones TH. Testosterone: a vascular hormone in health and disease, J Endocrinol., 2013; 217(3): R47-R71.

Liang Y, Liu R, Du S, Qiu C. Trends in incidence of hypertension in Chinese adults, 1991-2009: the China Health and Nutrition Survey. Int J Cardiol., 2014; 175(1): 96-101

Lloyd-Sherlock P, Beard J, Minicuci N, Ebrahim S, Chatterji S. Hypertension among older adults in low- and middle-income countries: prevalence, awareness and control, Int J Epidemiol., 2014; 43(1):116-28

Low BS, Choi SB, Abdul Wahab H, Das PK, Chan KL. Eurycomanone, the major quassinoid in Eurycoma longifolia root extract increases spermatogenesis by inhibiting the activity of phosphodiesterase and aromatase in steroidogenesis.J Ethnopharmacol., 2013; 149(1):201-7

Mohd Ridzuan MA, Sow A, Noor Rain A, Mohd Ilham A, Zakiah I. Eurycoma longifolia extract-artemisinin combination: parasitemia suppression of Plasmodium yoelii-infected mice. , Trop Biomed. 2007; 24(1):111-8.

Morris PD, Channer KS. Testosterone and cardiovascular disease in men. Asian J Androl. 2012; 14(3): 428-35.

Oteh M, Azarisman SM, Azreen SA, Jamaluddin AR, Aszrin A, Ting CK, Shaiful Bahri I. Institutional hypertension control in Malaysia: a multicenter study focusing on gender and cardiovascular risk factor profile difference.Hypertens Res. 2011; 34(3): 319-24.

Passeri E, Bonomi M, Dangelo F, Persani L, Corbetta S. Wasting syndrome with deep bradycardia as presenting manifestation of long-standing severe male hypogonadotropic hypogonadism: a case series. BMC Endocr Disord,. 2014; 14 :78.
Rampal L, Rampal S, Azhar MZ, Rahman AR. Prevalence, awareness, treatment and control of hypertension in Malaysia: a national study of 16,440 subjects.', Public Health,. 2008; 122(1): 11-8.

Rashid A, Azizah A. Prevalence of hypertension among the elderly Malays living in rural Malaysia., Australas Med J,. 2011; 4(6): 283-90.

Saadiah Abdul Razak H, Shuid AN, Naina Mohamed I. Combined Effects of Eurycoma longifolia and Testosterone on AndrogenDeficient Osteoporosis in a Male Rat Model, Evid Based Complement Alternat Med,. 2012; 2012: 872406

Sandberg, K. and Ji, H. Sex differences in primary hypertension,. Biol Sex Differ,. 2012:1-21.

Tada H, Yasuda F, Otani K, Doteuchi M, Ishihara Y, Shiro M. New antiulcer quassinoids from Eurycoma longifolia. Eur J Med Chem,. 1991;26:345-9

Tajul Ariff AS, Soelaiman IN, Pramanik J, Shuid AN. Effects of Eurycoma longifolia on testosterone level and bone structure in an aged orchidectomised rat model', Evid Based Complement Alternat Med, 2012;2012

Talbott SM, Talbott JA, George A, Pugh M. Effect of Tongkat Ali on stress hormones and psychological mood state in moderately stressed subjects.J Int Soc Sports Nutr,. 2013;,10(1):,28

Zanoli P, Zavatti M, Montanari C, Baraldi M. Influence of Eurycoma longifolia on the copulatory activity of sexually sluggish and impotent male rats, J Ethnopharmacol,. 2009;126(2):308-13.

\section{How to cite this article:}

Mokhtar RH, Al-Joufi F, Saxena AK, Al-Ani IM, Talib NA, KuZaifah N. The Effects of Eurycoma longifolia on Testosterone and Blood Pressure in High-Fat-Fed Animal Model. J App Pharm Sci, 2017; 7 (04): 119-124. 